\title{
On the number of primitive Pythagorean triangles
}

\author{
by \\ Wenguang Zhai (Jinan)
}

1. Introduction. A primitive Pythagorean triangle is a triple $(a, b, c)$ of natural numbers with

$$
a^{2}+b^{2}=c^{2}, \quad a \leq b, \quad \operatorname{gcd}(a, b, c)=1 .
$$

For a large real number $N$, let $P(N)$ denote the number of Pythagorean triangles with area less than $N$. Many authors studied the asymptotic behaviour of $P(N)$.

J. Lambek and L. Moser [3] proved that

$$
P(N)=c N^{1 / 2}+O\left(N^{1 / 3}\right)
$$

with $c=\left(2 \pi^{5}\right)^{-1 / 2} \Gamma^{2}(1 / 4)$.

R. E. Wild [10] proved that

$$
P(N)=c N^{1 / 2}-c^{\prime} N^{1 / 3}+R(N)
$$

with

$$
c^{\prime}=\frac{|\zeta(1 / 3)|\left(1+2^{-1 / 3}\right)}{\zeta(4 / 3)\left(1+4^{-1 / 3}\right)}, \quad R(N)=O\left(N^{1 / 4} \log N\right) .
$$

The best known bound for $R(N)$ depends on the estimation of

$$
M(x):=\sum_{n \leq x} \mu(n),
$$

where $\mu(n)$ is the Möbius function. Using the bound $M(x)=O\left(x e^{-\delta \log ^{1 / 2} x}\right)$, J. Duttlinger and W. Schwarz [1] proved (1.2) with

$$
R(N)=O\left(N^{1 / 4} e^{-\delta \log ^{1 / 2} N}\right) .
$$

The same argument with the bound $M(x)=O\left(x e^{-\delta \log ^{3 / 5} x \log \log ^{-1 / 5} x}\right)$ yields

$$
R(N)=O\left(N^{1 / 4} e^{-\delta \log ^{3 / 5} N \log \log ^{-1 / 5} N}\right) .
$$

2000 Mathematics Subject Classification: Primary 11P21.

Key words and phrases: primitive Pythagorean triangles, Riemann Hypothesis. 
Under the assumption of the Riemann Hypothesis (RH), J. Duttlinger and W. Schwarz [1] proved

$$
R(N)=O\left(N^{5 / 22+\varepsilon}\right)
$$

The exponent $5 / 22$ can be replaced by 137/604, 127/560, 37/164, 269/1238, as proved respectively by Menzer [4], Müller-Nowak-Menzer [6], Müller and Nowak [5], Nowak [7].

In this paper, we shall prove the following

THEOREM. If $R H$ is true, then

$$
R(N)=O\left(N^{127 / 616} \log ^{963 / 308} N\right) .
$$

Numerically, we have

$5 / 22=0.22727 \ldots, \quad 137 / 604=0.22681 \ldots, \quad 127 / 560=0.22678 \ldots$,

$37 / 164=0.2256 \ldots, \quad 269 / 1238=0.21728 \ldots, \quad 127 / 616=0.2061 \ldots$

Notations. $\mathbb{N}$ denotes the set of all natural numbers. Define

$$
\mathbb{N}^{\prime}=\sqrt{2} \mathbb{N}, \quad \mathbb{N}^{\prime \prime}=\sqrt{2} \mathbb{N}-\sqrt{2} / 2 .
$$

$\{t\}$ denotes the fractional part of $t$,

$$
\psi(t)=\{t\}-1 / 2, \quad \psi^{*}(t)=\{t\}^{2} / 2-\{t\} / 2+1 / 12 .
$$

$d \sim D$ means $D<d \leq 2 D$.

2. Reduction of the problem. J. Lambek and L. Moser [3] reduced the problem of calculating $P(N)$ to that of counting the number $L(N)$ of lattice points inside the planar domain

$$
\mathcal{D}=\left\{(x, y) \in \mathbb{R}^{2}: x y\left(x^{2}-y^{2}\right)<N, 0<y<x\right\}, \quad N \in \mathbb{R}^{+} .
$$

They established the formula

$$
P(N)=\sum_{k=0}^{\infty}(-1)^{k} L^{\prime}\left(\frac{N}{4^{k}}\right),
$$

where $L^{\prime}(N)$ is the number of primitive lattice points in $\mathcal{D}$. Thus the problem is reduced to estimating $L^{\prime}(N)$. By a usual device, we have

$$
L^{\prime}(N)=\sum_{d=1}^{\infty} \mu(d) L\left(\frac{N}{d^{4}}\right) .
$$

$L(N)$ can be written as

$$
L(N)=k N^{1 / 2}-k^{\prime} N^{1 / 3}+F(N),
$$

where

$$
k=\frac{\Gamma^{2}(1 / 4)}{4(2 \pi)^{1 / 2}}, \quad k^{\prime}=|\zeta(1 / 3)|\left(1+2^{-1 / 3}\right) .
$$


Suppose $1 \leq y<N^{1 / 4}$ is a parameter. We write

$$
L^{\prime}(N)=\sum_{d \leq y} \mu(d) L\left(\frac{N}{d^{4}}\right)+\sum_{d>y} \mu(d) L\left(\frac{N}{d^{4}}\right)=S_{1}+S_{2} .
$$

By (2.3), we have

$$
\begin{aligned}
S_{1} & =k N^{1 / 2} \sum_{d \leq y} \frac{\mu(d)}{d^{2}}-k^{\prime} N^{1 / 3} \sum_{d \leq y} \frac{\mu(d)}{d^{4 / 3}}+\sum_{d \leq y} \mu(d) F\left(\frac{N}{d^{4}}\right) \\
& =k N^{1 / 2} \sum_{d \leq y} \frac{\mu(d)}{d^{2}}-k^{\prime} N^{1 / 3} \sum_{d \leq y} \frac{\mu(d)}{d^{4 / 3}}+S_{1}^{*} .
\end{aligned}
$$

Now the problem is reduced to estimating $S_{1}^{*}$ and $S_{2}$.

3. Properties of $F(N)$. In this section we study the error term $F(N)$, which is very important in our proof.

For a large real number $N$, let

$$
Y=Y(N)=\sqrt{2} \sin (\pi / 8) N^{1 / 4} .
$$

For any $0<y<Y$, let $\xi(y)$ denote the unique solution of the equation

$$
x^{3} y-y^{3} x-N=0 .
$$

By Cardano's formula,

$$
\xi(y)=\left(\frac{N}{2 y}+\sqrt{D}\right)^{1 / 3}+\left(\frac{N}{2 y}-\sqrt{D}\right)^{1 / 3}, \quad D=\frac{1}{4}\left(\frac{N}{y}\right)^{2}-\frac{1}{27} y^{6} .
$$

In particular, $\xi(y)>y$ and $\xi(Y)=(\sqrt{2}+1) Y$. Let $\alpha=\sqrt{2}-1$. Define

$$
\begin{aligned}
& F_{1}(N)=\sum_{\substack{y<Y \\
y \in \mathbb{N}}} \psi(\xi(y)), \quad F_{2}(N)=\sum_{\substack{y<Y \\
y \in \mathbb{N}^{\prime}}} \psi\left(\frac{\xi(y)}{\sqrt{2}}\right), \\
& F_{3}(N)=\sum_{\substack{y<Y \\
y \in \mathbb{N}^{\prime \prime}}} \psi\left(\frac{\xi(y)}{\sqrt{2}}+\frac{1}{2}\right) .
\end{aligned}
$$

J. Duttlinger and W. Schwarz [1] proved that

$$
F(N)=-F_{1}(N)-F_{2}(N)-F_{3}(N)+O\left(N^{2 / 15}\right) .
$$

We first prove the following

Lemma 3.1. We have

$$
F(N)=-F_{1}(N)-F_{2}(N)-F_{3}(N)+O\left(\log ^{2} N\right) .
$$

Proof. We begin with formula (2.2) of [1], which reads

$$
L(N)=H_{1}-H_{2}-F_{1}-F_{2}-F_{3}+G,
$$


where

$$
\begin{aligned}
H_{1} & =\sum_{\substack{y<Y \\
y \in \mathbb{N}}} \xi(y)+\frac{1}{\sqrt{2}} \sum_{\substack{y<Y \\
y \in \mathbb{N}^{\prime}}} \xi(y)+\frac{1}{\sqrt{2}} \sum_{\substack{y<Y \\
y \in \mathbb{N}^{\prime \prime}}} \xi(y), \\
H_{2} & =\sum_{\substack{y<Y \\
y \in \mathbb{N}}} \alpha^{-1} y+\frac{1}{\sqrt{2}} \sum_{\substack{y<Y \\
y \in \mathbb{N}^{\prime}}} \alpha^{-1} y+\frac{1}{\sqrt{2}} \sum_{\substack{y<Y \\
y \in \mathbb{N}^{\prime \prime}}} \alpha^{-1} y, \\
G & =\sum_{\substack{y<Y \\
y \in \mathbb{N}}} \psi\left(\alpha^{-1} y\right)+\sum_{\substack{y<Y \\
y \in \mathbb{N}^{\prime}}} \psi\left(\frac{\alpha^{-1} y}{\sqrt{2}}\right)+\sum_{\substack{y<Y \\
y \in \mathbb{N}^{\prime \prime}}} \psi\left(\frac{\alpha^{-1} y}{\sqrt{2}}+\frac{1}{2}\right) .
\end{aligned}
$$

In order to estimate $H_{1}$, we use the following Euler-Maclaurin formula: suppose $f(u)$ is three times continuously differentiable on $[1, U]$; then

$$
\begin{aligned}
\sum_{1 \leq n \leq U} f(n)= & \int_{1}^{U} f(u) d u-f(U) \psi(U)+f(1) / 2+\psi^{*}(U) f^{\prime}(U) \\
& -\psi^{*}(1) f^{\prime}(1)-\int_{1}^{U} \psi^{*}(u) f^{\prime \prime}(u) d u .
\end{aligned}
$$

This formula can be found in [8, Chapter 2].

We first evaluate the sum $\sum_{y<Y, y \in \mathbb{N}} \xi(y)$. By (3.3) we have

$$
\begin{aligned}
\sum_{\substack{y<Y \\
y \in \mathbb{N}}} \xi(y)= & \int_{1}^{Y} \xi(u) d u-\xi(Y) \psi(Y)+\xi(1) / 2+\psi^{*}(Y) \xi^{\prime}(Y) \\
& -\psi^{*}(1) \xi^{\prime}(1)-\int_{1}^{Y} \psi^{*}(u) \xi^{\prime \prime}(u) d u .
\end{aligned}
$$

Müller, Nowak and Menzer [6] proved that for $1 \leq u \leq Y$, the estimate

$$
\left|\xi^{(r)}(u)\right| \asymp N^{1 / 3} u^{-1 / 3-r}
$$

holds for $r=1,2,3$. This implies that $\xi^{\prime \prime}(u)$ is monotone and $\xi^{\prime \prime}(u) \ll 1$ for $u \gg N^{1 / 7}, \xi^{\prime}(Y) \ll 1$.

We write

$$
\int_{1}^{Y} \psi^{*}(u) \xi^{\prime \prime}(u) d u=\int_{1}^{N^{1 / 7}} \psi^{*}(u) \xi^{\prime \prime}(u) d u+\int_{N^{1 / 7}}^{Y} \psi^{*}(u) \xi^{\prime \prime}(u) d u .
$$

By partial integration, we get

$$
\int_{N^{1 / 7}}^{Y} \psi^{*}(u) \xi^{\prime \prime}(u) d u \ll 1
$$


For $0<u \leq N^{1 / 7}$, it is easy to check that

$$
\begin{aligned}
\xi(u) & =\frac{N^{1 / 3}}{u^{1 / 3}}+O\left(\frac{u^{7 / 3}}{N^{1 / 3}}\right), \\
\xi^{\prime}(u) & =-\frac{1}{3} \cdot \frac{N^{1 / 3}}{u^{4 / 3}}+O\left(\frac{u^{4 / 3}}{N^{1 / 3}}\right), \\
\xi^{\prime \prime}(u) & =\frac{4}{9} \cdot \frac{N^{1 / 3}}{u^{7 / 3}}+O\left(\frac{u^{1 / 3}}{N^{1 / 3}}\right) .
\end{aligned}
$$

So we get

$$
\begin{aligned}
& \int_{1}^{N^{1 / 7}} \psi^{*}(u) \xi^{\prime \prime}(u) d u \\
& =\frac{4 N^{1 / 3}}{9} \int_{1}^{N^{1 / 7}} \psi^{*}(u) u^{-7 / 3} d u+O\left(N^{-1 / 3} \int_{1}^{N^{1 / 7}} u^{1 / 3} d u\right) \\
& =\frac{4 N^{1 / 3}}{9} \int_{1}^{\infty} \psi^{*}(u) u^{-7 / 3} d u-\frac{4 N^{1 / 3}}{9} \int_{N^{1 / 7}}^{\infty} \psi^{*}(u) u^{-7 / 3} d u+O(1) \\
& =c_{0} N^{1 / 3}+O(1),
\end{aligned}
$$

with

$$
\begin{aligned}
c_{0} & =\frac{4}{9} \int_{1}^{\infty} \psi^{*}(u) u^{-7 / 3} d u=\zeta(1 / 3)+1+1 / 36, \\
\xi(1) / 2 & =N^{1 / 3} / 2+O(1), \quad \psi^{*}(1) \xi^{\prime}(1)=-N^{1 / 3} / 36+O(1) .
\end{aligned}
$$

From (3.4)-(3.11) we get

$$
\sum_{\substack{y<Y \\ y \in \mathbb{N}}} \xi(y)=\int_{1}^{Y} \xi(u) d u+c_{1} N^{1 / 3}-\xi(Y) \psi(Y)+O(1),
$$

where $c_{1}=1 / 2+1 / 36-c_{0}$.

Similarly we get

$$
\begin{aligned}
& \frac{1}{\sqrt{2}} \sum_{\substack{y<Y \\
y \in \mathbb{N}^{\prime}}} \xi(y)=\frac{1}{\sqrt{2}} \sum_{\substack{m<Y / \sqrt{2} \\
m \in \mathbb{N}}} \xi(\sqrt{2} m) \\
& \quad=\frac{1}{\sqrt{2}} \int_{1}^{Y / \sqrt{2}} \xi(\sqrt{2} u) d u+c_{2}^{\prime} N^{1 / 3}-\frac{\xi(Y)}{\sqrt{2}} \psi\left(\frac{Y}{\sqrt{2}}\right)+O(1) \\
& =\frac{1}{2} \int_{\sqrt{2}}^{Y} \xi(u) d u+c_{2}^{\prime} N^{1 / 3}-\frac{\xi(Y)}{\sqrt{2}} \psi\left(\frac{Y}{\sqrt{2}}\right)+O(1)
\end{aligned}
$$




$$
\begin{aligned}
& =\frac{1}{2} \int_{1}^{Y} \xi(u) d u-\frac{1}{2} \int_{1}^{\sqrt{2}} \xi(u) d u+c_{2}^{\prime} N^{1 / 3}-\frac{\xi(Y)}{\sqrt{2}} \psi\left(\frac{Y}{\sqrt{2}}\right)+O(1) \\
& =\frac{1}{2} \int_{1}^{Y} \xi(u) d u+c_{2} N^{1 / 3}-\frac{\xi(Y)}{\sqrt{2}} \psi\left(\frac{Y}{\sqrt{2}}\right)+O(1), \\
& 3.14) \quad \frac{1}{\sqrt{2}} \sum_{y<Y} \xi(y)=\frac{1}{\sqrt{2}} \sum_{m<Y / \sqrt{2}+1 / 2} \xi\left(\sqrt{2} m-\frac{\sqrt{2}}{2}\right) \\
& =\frac{1}{\sqrt{2}} \int_{y \in \mathbb{N}}^{Y / \sqrt{2}+1 / 2} \xi\left(\sqrt{2} u-\frac{\sqrt{2}}{2}\right) d u+c_{3}^{\prime} N^{1 / 3}-\frac{\xi(Y)}{\sqrt{2}} \psi\left(\frac{Y}{\sqrt{2}}+\frac{1}{2}\right)+O(1) \\
& =\frac{1}{2} \int_{\sqrt{2} / 2}^{Y} \xi(u) d u+c_{3}^{\prime} N^{1 / 3}-\frac{\xi(Y)}{\sqrt{2}} \psi\left(\frac{Y}{\sqrt{2}}+\frac{1}{2}\right)+O(1) \\
& =\frac{1}{2} \int_{1}^{Y} \xi(u) d u+\frac{1}{2} \int_{\sqrt{2} / 2} \int_{1}(u) d u+c_{3}^{\prime} N^{1 / 3}-\frac{\xi(Y)}{\sqrt{2}} \psi\left(\frac{Y}{\sqrt{2}}+\frac{1}{2}\right)+O(1) \\
& =\frac{1}{2} \int_{1}^{Y} \xi(u) d u+c_{3} N^{1 / 3}-\frac{\xi(Y)}{\sqrt{2}} \psi\left(\frac{Y}{\sqrt{2}}+\frac{1}{2}\right)+O(1),
\end{aligned}
$$

where $c_{2}, c_{2}^{\prime}, c_{3}, c_{3}^{\prime}$ are constants.

From (3.12)-(3.14) we get

$$
\begin{aligned}
H_{1}= & 2 \int_{1}^{Y} \xi(u) d u-k^{\prime} N^{1 / 3}-\xi(Y) \psi(Y) \\
& -\frac{\xi(Y)}{\sqrt{2}} \psi\left(\frac{Y}{\sqrt{2}}\right)-\frac{\xi(Y)}{\sqrt{2}} \psi\left(\frac{Y}{\sqrt{2}}+\frac{1}{2}\right)+O(1) .
\end{aligned}
$$

For $\int_{1}^{Y} \xi(u) d u$, we have

$$
\int_{1}^{Y} \xi(u) d u=\frac{1}{4} N^{1 / 2} \frac{\Gamma^{2}(1 / 4)}{2(2 \pi)^{1 / 2}}+\frac{\alpha^{-1} Y^{2}}{2} .
$$

This is contained in [1].

It is easy to check that

$$
\begin{aligned}
H_{2}= & \alpha^{-1} Y^{2}-\alpha^{-1} \xi(Y) Y \\
& -\alpha^{-1} \frac{Y}{\sqrt{2}} \psi\left(\frac{Y}{\sqrt{2}}\right)-\alpha^{-1} \frac{Y}{\sqrt{2}} \psi\left(\frac{Y}{\sqrt{2}}+\frac{1}{2}\right)+O(1)
\end{aligned}
$$




$$
\begin{aligned}
= & \alpha^{-1} Y^{2}-\xi(Y) \psi(Y)-\frac{\xi(Y)}{\sqrt{2}} \psi\left(\frac{Y}{\sqrt{2}}\right) \\
& -\frac{\xi(Y)}{\sqrt{2}} \psi\left(\frac{Y}{\sqrt{2}}+\frac{1}{2}\right)+O(1),
\end{aligned}
$$

if we notice $\xi(Y)=(\sqrt{2}+1) Y$.

For $G$, we have

$$
G=O\left(\log ^{2} N\right)
$$

This is formula (3.8) of [1]. Now Lemma 3.1 follows from (3.2), (3.15)(3.18).

Lemma 3.2. Suppose $T \geq 10$. Then

$$
\int_{1}^{T}|F(u)|^{2} d u \ll T^{5 / 4} \log ^{4} T .
$$

Proof. By Lemma 3.1, it suffices to prove that

$$
\int_{T}^{T+\varepsilon T}\left|F_{i}(u)\right|^{2} d u \ll T^{5 / 4} \log ^{4} T \quad(i=1,2,3)
$$

for a small $\varepsilon>0$. We only consider the case $i=1$. The proofs for the other two cases are the same.

We write

$$
\begin{aligned}
F_{1}(u) & =\sum_{\substack{y<\sqrt{2} \sin (\pi / 8) u^{1 / 4} \\
y \in \mathbb{N}}} \psi(\xi(y, u)) \\
& =\sum_{j=1}^{J} \sum_{\substack{y \sim \sqrt{2} \sin (\pi / 8) \\
y \in \mathbb{N}}} \psi(\xi(y, u))+O\left(T^{1 / 4}\right), \\
y 2^{j} & \psi
\end{aligned}
$$

where $J=[\log T /(8 \log 2)]$, and $\xi(y, u)$ is the unique solution of the equation

$$
\xi^{3} y-y^{3} \xi=u
$$

where

$$
(u, y) \in\left\{(u, y): T \leq u \leq T+\varepsilon T, 0<y<\sqrt{2} \sin (\pi / 8) u^{1 / 4}\right\} .
$$

Thus

$$
F_{1}(u) \ll\left|\sum_{\substack{y \sim \sqrt{2} \sin (\pi / 8) u^{1 / 4} / 2^{j_{0}} \\ y \in \mathbb{N}}} \psi(\xi(y, u))\right| \log T+T^{1 / 8}
$$

for some $1 \leq j_{0} \leq J$ 
We use the following expression (3.21) of $\psi(t)$ to transform the above sum into the exponential sum: for any $H_{0} \geq 2$,

$$
\psi(t)=\sum_{1 \leq|h| \leq H_{0}} a(h) e(h t)+O\left(\sum_{1 \leq h \leq H_{0}} b(h) e(h t)\right)+O\left(1 / H_{0}\right)
$$

with $a(h) \ll 1 /|h|$ and $b(h) \ll 1 / H_{0}$. This is due to Vaaler [9].

Take $H_{0}=T^{1 / 4} 2^{-j_{0}}$ in (3.21). We get

$$
F_{1}(u) \ll \sum_{1 \leq h \leq H_{0}} \frac{1}{h}\left|\sum_{\substack{y \sim \sqrt{2} \sin (\pi / 8) u^{1 / 4} / 2^{j_{0}} \\ y \in \mathbb{N}}} e(\xi(y, u))\right| \log T+T^{1 / 8} .
$$

By Cauchy's inequality,

$$
\begin{aligned}
& \left|F_{1}(u)\right|^{2} \\
& \quad \ll\left(\sum_{1 \leq h \leq H_{0}} \frac{1}{h^{1 / 2}} \cdot \frac{1}{h^{1 / 2}}\left|\sum_{\substack{y \sim \sqrt{2} \sin (\pi / 8) u^{1 / 4} / 2^{j_{0}} \\
y \in \mathbb{N}}} e(h \xi(y, u))\right|\right)^{2} \log ^{2} T+T^{1 / 4} \\
& \quad \ll \sum_{1 \leq h \leq H_{0}} \frac{1}{h}\left|\sum_{\substack { y \sim \sqrt{2} \\
\begin{subarray}{c}{\sin (\pi / 8) u^{1 / 4} / 2^{j 0} \\
y \in \mathbb{N}{ y \sim \sqrt { 2 } \\
\begin{subarray} { c } { \operatorname { s i n } ( \pi / 8 ) u ^ { 1 / 4 } / 2 ^ { j 0 } \\
y \in \mathbb { N } } }\end{subarray}} e(h \xi(y, u))\right|^{2} \log ^{3} T+T^{1 / 4} .
\end{aligned}
$$

Thus

$$
\begin{aligned}
& \text { (3.22) } \log ^{-3} T \int_{T}^{T+\varepsilon T}\left|F_{1}(u)\right|^{2} d u \\
& \ll \sum_{1 \leq h \leq H_{0}} \frac{1}{h} \int_{T}^{T+\varepsilon T}\left|\sum_{\substack{y \sim \sqrt{2} \sin (\pi / 8) u^{1 / 4} / 2^{j 0} \\
y \in \mathbb{N}}} e(h \xi(y, u))\right|^{2} d u+T^{5 / 4}
\end{aligned}
$$$$
=\sum_{1 \leq h \leq H_{0}} \frac{1}{h} \int_{T}^{T+\varepsilon T} \sum_{y_{1}, y_{2}} e\left(h \xi\left(y_{1}, u\right)-h \xi\left(y_{2}, u\right)\right) d u+T^{5 / 4}
$$$$
\ll \varepsilon T N_{0} \sum_{1 \leq h \leq H_{0}} \frac{1}{h}+\sum_{1 \leq h \leq H_{0}} \frac{1}{h}\left|\int_{T}^{T+\varepsilon T} \sum_{y_{1} \neq y_{2}} e\left(h \xi\left(y_{1}, u\right)-h \xi\left(y_{2}, u\right)\right) d u\right|+T^{5 / 4}
$$$$
\ll \sum_{1 \leq h \leq H_{0}} \frac{1}{h} \sum_{\substack{y_{1} \neq y_{2} \\ y_{i} \sim N_{0}, y_{i} \in \mathbb{N}}}\left|\int_{I\left(y_{1}, y_{2}\right)} e\left(h \xi\left(y_{1}, u\right)-h \xi\left(y_{2}, u\right)\right) d u\right|+T^{5 / 4} \log T,
$$

where $I\left(y_{1}, y_{2}\right)$ is a subinterval of $[T, T+\varepsilon T], N_{0}=\sqrt{2} \sin (\pi / 8) T^{1 / 4} / 2^{j_{0}}$.

In order to estimate the last integral in (3.22), we need the following well known result (see, for example, [8, Chapter 21]): suppose $f(t)$ is a real- 
valued function defined on $[a, b]$ such that $f^{\prime}(t)$ is monotone on $[a, b]$ and $\left|f^{\prime}(t)\right| \gg \delta>0$; then

$$
\int_{a}^{b} e(f(t)) d t \ll \delta^{-1} .
$$

Let $D(u)=h \xi\left(y_{1}, u\right)-h \xi\left(y_{2}, u\right)$. We need to compute $D_{u}$.

From the equation $\xi^{3} y-y^{3} \xi=u$ it is easy to get

$$
\xi_{u}=\frac{1}{3 \xi^{2} y-y^{3}}, \quad \xi_{y}=\frac{3 y^{2} \xi-\xi^{3}}{3 \xi^{2} y-y^{3}} .
$$

Thus

$$
D_{u}=h\left(\xi_{u}\left(y_{1}, u\right)-\xi_{u}\left(y_{2}, u\right)\right)=h\left(\frac{1}{3 \xi\left(y_{1}, u\right)^{2} y_{1}-y_{1}^{3}}-\frac{1}{3 \xi\left(y_{2}, u\right)^{2} y_{2}-y_{2}^{3}}\right) .
$$

For fixed $u \in[T, T+\varepsilon T]$, let $D_{1}(y)=3 \xi(y, u)^{2} y-y^{3}$. By Lagrange's theorem, there exists a $y_{0} \sim N_{0}$ such that

$$
D_{u}=h \xi_{u y}\left(y_{0}, u\right)\left(y_{1}-y_{2}\right) .
$$

It is easy to show that

$$
\begin{aligned}
\xi_{u y} & =-\frac{D_{1}^{\prime}(y)}{D_{1}^{2}(y)}=-\frac{3}{D_{1}^{2}(y)}\left(2 \xi y \xi_{y}+\xi^{2}-y^{2}\right) \\
& =-\frac{3 y\left(\xi^{2}+y^{2}\right)^{2}}{D_{1}^{3}(y)}=-\frac{3\left(\xi^{2}+y^{2}\right)^{2}}{y^{2}\left(3 \xi^{2}-y^{2}\right)^{3}},
\end{aligned}
$$

which implies that (notice $\xi \asymp N^{1 / 3} y^{-1 / 3}$ )

$$
D_{u} \gg \frac{h\left|y_{1}-y_{2}\right|}{T^{2 / 3} N_{0}^{4 / 3}} .
$$

Thus by (3.23) we get

$$
\int_{I\left(y_{1}, y_{2}\right)} e\left(h \xi\left(y_{1}, u\right)-h \xi\left(y_{2}, u\right)\right) d u \ll \frac{T^{2 / 3} N_{0}^{4 / 3}}{h\left|y_{1}-y_{2}\right|},
$$

which leads to

$$
\begin{aligned}
& \log ^{-3} T \int_{T}^{T+\varepsilon T}\left|F_{1}(u)\right|^{2} d u \\
& \ll \sum_{1 \leq h \leq H_{0}} \frac{1}{h} \sum_{\substack{y_{1} \neq y_{2} \\
y_{i} \sim N_{0}, y_{i} \in \mathbb{N}}} \frac{T^{2 / 3} N_{0}^{4 / 3}}{h\left|y_{1}-y_{2}\right|}+T^{5 / 4} \log T \ll T^{5 / 4} \log T,
\end{aligned}
$$

if we notice $N_{0} \ll T^{1 / 4}$. Thus, (3.19) holds for $i=1$. In the same way we can prove the cases $i=2,3$. 
4. Estimation of $S_{1}^{*}$. In this section we shall estimate $S_{1}^{*}$ by an argument similar to one of Huxley and Nowak [2].

Since $L(N)$ is increasing, we get $L(u) \geq L(v)$ for any $u \geq v \geq 1$, which implies that

$$
F(u)-F(v) \geq k\left(v^{1 / 2}-u^{1 / 2}\right)-k^{\prime}\left(v^{1 / 3}-u^{1 / 3}\right)
$$

So, if $V$ is some positive number and $t>1$ is a value for which $|F(t)| \geq V$, there exists an interval $I$ of length $\geq t^{1 / 2} V / 100$ containing $t$ such that

$$
|F(u)| \geq V / 3
$$

for all $u \in I$.

For positive real parameters $D \ll N^{1 / 4}$ and $V \geq\left(N / D^{4}\right)^{1 / 8}$, let

$$
\begin{aligned}
\mathcal{M}(D, V) & =\left\{d \in \mathbb{N}: D<d \leq 2 D, V \leq\left|F\left(N / d^{4}\right)\right| \leq 2 V\right\}, \\
\mathcal{R}(D, V) & =\# \mathcal{M}(D, V) .
\end{aligned}
$$

For any $d \in \mathcal{M}(D, V)$, by the above considerations, there exists an interval

$$
J \subset\left[\frac{N}{(d+1 / 2)^{4}}, \frac{N}{(d-1 / 2)^{4}}\right]
$$

of length $|J| \gg \min \left(N^{1 / 2} V / D^{2}, N / D^{5}\right)$ such that

$$
|F(u)| \geq V / 3
$$

for all $u \in J$. By Lemma 3.2 we have

$$
V^{2} \mathcal{R}(D, V) \min \left(\frac{N^{1 / 2} V}{D^{2}}, \frac{N}{D^{5}}\right) \ll \int_{1}^{N /(D-1 / 2)^{4}}|F(u)|^{2} d u \ll \frac{N^{5 / 4}}{D^{5}} \log ^{4} N
$$

Thus we get

$$
\mathcal{R}(D, V) \ll\left(\frac{N^{3 / 4}}{D^{3} V^{3}}+\frac{N^{1 / 4}}{V^{2}}\right) \log ^{4} N .
$$

By a familiar device, we get

$$
\begin{aligned}
& \sum_{D<d \leq 2 D}\left|F\left(\frac{N}{d^{4}}\right)\right| \\
\ll & N^{1 / 8} D^{1 / 2}+\sum_{V=2^{j} \gg N^{1 / 8} D^{-1 / 2}, j \in \mathbb{N}} V \min (D, \mathcal{R}(D, V)) \\
\ll & N^{1 / 8} D^{1 / 2}+\sum_{V=2^{j} \gg N^{1 / 8} D^{-1 / 2}, j \in \mathbb{N}} \min \left(D V, \frac{N^{3 / 4}}{D^{3} V^{2}}+\frac{N^{1 / 4}}{V}\right) \log ^{4} N \\
\ll & N^{1 / 4} D^{-1 / 3} \log ^{4} N+N^{1 / 8} D^{1 / 2} \log ^{4} N .
\end{aligned}
$$


For $F(u)$, we have the estimate (see [7, p. 176])

$$
F(u) \ll u^{23 / 146} \log ^{315 / 146} u,
$$

which implies

$$
\sum_{D<d \leq 2 D}\left|F\left(\frac{N}{d^{4}}\right)\right| \ll N^{23 / 146} D^{54 / 146} \log ^{315 / 146} N .
$$

Suppose $1<y_{1}<y$ is a parameter. From (4.2) we have

$$
\sum_{y_{1}<d \leq y}\left|F\left(\frac{N}{d^{4}}\right)\right| \ll N^{1 / 4} y_{1}^{-1 / 3} \log ^{4} N+N^{1 / 8} y^{1 / 2} \log ^{4} N
$$

From (4.4) we get

$$
\sum_{d \leq y_{1}}\left|F\left(\frac{N}{d^{4}}\right)\right| \ll N^{23 / 146} y_{1}^{54 / 146} \log ^{315 / 146} N .
$$

Combining (4.5) and (4.6) we get the estimate

$$
\begin{aligned}
S_{1}^{*}= & \sum_{d \leq y} \mu(d) F\left(\frac{N}{d^{4}}\right) \ll \sum_{d \leq y_{1}}\left|F\left(\frac{N}{d^{4}}\right)\right|+\sum_{y_{1}<d \leq y}\left|F\left(\frac{N}{d^{4}}\right)\right| \\
\ll & N^{23 / 146} y_{1}^{54 / 146} \log ^{315 / 146} N \\
& \quad+N^{1 / 4} y_{1}^{-1 / 3} \log ^{4} N+N^{1 / 8} y^{1 / 2} \log ^{4} N \\
\ll & N^{127 / 616} \log ^{963 / 308} N+N^{1 / 8} y^{1 / 2} \log ^{4} N
\end{aligned}
$$

on taking $y_{1}=N^{81 / 616} \log ^{807 / 308} N$. The estimate (4.7) is true for any $1 \leq$ $y \ll N^{1 / 4}$.

5. Estimation of $S_{2}$ and proof of the Theorem. In this section we shall estimate $S_{2}$ and give the proof of the Theorem. We first study the properties of

$$
Z(s)=\sum_{n=1}^{\infty} r(n) n^{-s}, \quad \Re s>1 / 2,
$$

where $r(n)$ is defined by

$$
r(n)=\sum_{\substack{n=x y\left(x^{2}-y^{2}\right) \\ x, y \in \mathbb{N}}} 1 .
$$

LEMma 5.1. We have the following estimates:

$$
\sum_{n=1}^{\infty} \frac{r^{2}(n)}{n^{\sigma}} \ll 1, \quad \sum_{n=1}^{\infty} \frac{r(n)}{n^{\sigma}} \ll 1, \quad \sigma>1 / 2 ;
$$




$$
\sum_{n \leq x} \frac{r(n)}{n^{1 / 2}} \ll \log x, \quad \sum_{n \leq x} \frac{r(n)}{n^{\sigma}} \ll x^{1 / 2-\sigma}, \quad 0<\sigma<1 / 2 .
$$

Proof. These estimates follow from (2.3) by partial summation.

LEMMA 5.2. $Z(s)$ has the following properties:

(1) $Z(s)$ has an analytic continuation to $\sigma>1 / 8$ with two simple poles at $s=1 / 2$ and $s=1 / 3$.

(2) We have

$$
Z(\sigma+i t) \ll \min \left(\log |t|, \frac{1}{\sigma-1 / 2}\right), \quad \sigma \geq 1 / 2,|t| \geq 2 .
$$

(3) The estimate

$$
Z(\sigma+i t) \ll|t|^{8(1 / 2-\sigma) / 3} \log |t|
$$

holds uniformly for $1 / 8<\sigma_{1} \leq \sigma \leq 1 / 2,|t| \geq 2$.

(4) For any real parameter $T \geq 10$, we have

$$
\int_{T}^{2 T}|Z(24 / 73+i t)|^{2} d t \ll T \log ^{7} T .
$$

Proof. Suppose $X \geq 2$ is a parameter. For $\sigma>1 / 2$, by Stieltjes integration we get

$$
\begin{aligned}
& Z(s)=\sum_{n \leq X} \frac{r(n)}{n^{s}}+\int_{X}^{\infty} \omega^{-s} d L(\omega) \\
= & \sum_{n \leq X} \frac{r(n)}{n^{s}}+\int_{X}^{\infty} \omega^{-s} d\left(k \omega^{1 / 2}-k^{\prime} \omega^{1 / 3}+F(\omega)\right) \\
= & \sum_{n \leq X} \frac{r(n)}{n^{s}}+\frac{k}{2} \cdot \frac{X^{1 / 2-s}}{s-1 / 2}-\frac{k^{\prime}}{3} \cdot \frac{X^{1 / 3-s}}{s-1 / 3}-X^{-s} F(X)+s \int_{X}^{\infty} \frac{F(\omega)}{\omega^{s+1}} d \omega .
\end{aligned}
$$

From Lemma 3.2 we get

$$
\int_{1}^{M}|F(u)| d u \ll M^{9 / 8} \log ^{2} M
$$

This shows that the integration on the right side of (5.1) is absolutely convergent for $\sigma>1 / 8$. So the first assertion of Lemma 5.2 follows.

The second assertion follows from (5.1) and Lemma 5.1.

Suppose $1 / 8<\sigma_{1}<1 / 2$. Then from Lemma 5.1 and (5.1) we get

$$
Z\left(\sigma_{1}+i t\right) \ll X^{1 / 2-\sigma_{1}}+|t| X^{1 / 8-\sigma} \ll|t|^{8(1 / 2-\sigma) / 3}
$$

by choosing $X=|t|^{8 / 3}$. Now the third assertion of Lemma 5.2 follows from the Pragmén-Lindelöf argument. 
Now we prove assertion (4). We always suppose $s=\sigma+i t, T \leq t \leq 2 T$, $24 / 73 \leq \sigma<1 / 2$, and $T \leq X \leq T^{3}$ is a parameter to be determined. From (5.1) we have

$$
\int_{T}^{2 T}|Z(\sigma+i t)|^{2} d t \ll W_{1}+T^{2} W_{2}+T^{-1} X^{1-2 \sigma}+T,
$$

where

$$
W_{1}=\int_{T}^{2 T}\left|\sum_{n \leq X} \frac{r(n)}{n^{\sigma+i t}}\right|^{2} d t, \quad W_{2}=\int_{T}^{2 T}\left|\int_{X}^{\infty} \frac{F(\omega)}{\omega^{\sigma+i t+1}} d \omega\right|^{2} d t .
$$

We first estimate $W_{1}$. Squaring, integrating and then using Lemma 5.1 we get

$$
\begin{aligned}
W_{1} & =\int_{T}^{2 T} \sum_{m, n \leq X} \frac{r(m) r(n)}{(m n)^{\sigma}}\left(\frac{m}{n}\right)^{i t} d t \\
& \ll T \sum_{n \leq X} \frac{r^{2}(n)}{n^{2 \sigma}}+\sum_{m \neq n} \frac{r(m) r(n)}{(m n)^{\sigma}} \min \left(T, \frac{1}{\left|\log \frac{m}{n}\right|}\right) \\
& \ll T+\sum_{m<n \leq X} \frac{r(m) r(n)}{(m n)^{\sigma}} \min \left(T, \frac{1}{\log \frac{n}{m}}\right) \\
& =T+\Sigma_{1}+\Sigma_{2}+\Sigma_{3},
\end{aligned}
$$

where

$$
\begin{aligned}
& \Sigma_{1}=T \sum_{m \leq X} \sum_{m<n \leq e^{1 / T} m} \frac{r(m) r(n)}{(m n)^{\sigma}}, \\
& \Sigma_{2}=\sum_{m \leq X} \sum_{e^{1 / T}} \frac{r(m) r(n)}{(m n)^{\sigma}} \cdot \frac{1}{\log \frac{n}{m}}, \\
& \Sigma_{3}=\sum_{m \leq X} \sum_{n>2 m} \frac{r(m) r(n)}{(m n)^{\sigma}} \cdot \frac{1}{\log \frac{n}{m}} .
\end{aligned}
$$

By Lemma 5.1 again we get

$$
\Sigma_{3} \ll\left(\sum_{m \leq X} \frac{r(m)}{m^{\sigma}}\right)^{2} \ll X^{1-2 \sigma} .
$$

For $\Sigma_{1}$, by Lemma 5.1 and (4.3) we get

$$
\begin{aligned}
\Sigma_{1} & \ll T \sum_{m \leq X} \frac{r(m)}{m^{2 \sigma}} \sum_{m<n \leq e^{1 / T} m} r(n) \\
& =T \sum_{m \leq X} \frac{r(m)}{m^{2 \sigma}}\left(L\left(e^{1 / T} m\right)-L(m)\right)
\end{aligned}
$$


400

$$
\begin{aligned}
& =T \sum_{m \leq X} \frac{r(m)}{m^{2 \sigma}}\left(k\left(e^{1 /(2 T)} m^{1 / 2}-m^{1 / 2}\right)-k^{\prime}\left(e^{1 /(3 T)} m^{1 / 3}-m^{1 / 3}\right)\right) \\
& \quad+T \sum_{m \leq X} \frac{r(m)}{m^{2 \sigma}}\left(F\left(e^{1 / T} m\right)-F(m)\right) \\
& \ll X^{1-2 \sigma}+T \log ^{4} T
\end{aligned}
$$

if we notice $\sigma \geq 24 / 73$.

It remains to estimate $\Sigma_{2}$. Let $n=m+r$ and notice

$$
\frac{1}{\log (n / m)}=\frac{1}{\log (1+r / m)} \ll m / r \text {. }
$$

Hence we get

$$
\Sigma_{2} \ll \sum_{m \leq X} \frac{r(m)}{m^{2 \sigma-1}} \Sigma_{4} \frac{r(m+r)}{r},
$$

where $\Sigma_{4}$ sums over $\max \left(1, m\left(e^{1 / T}-1\right)\right) \leq r \leq m$.

By a splitting argument and (4.3) we get

$$
\begin{aligned}
\Sigma_{4} & \ll \log m \cdot \max _{a \ll m} \sum_{a<r \leq 2 a} \frac{r(m+r)}{r} \\
& \ll \log m \cdot \max _{a \ll m} \frac{1}{a} \sum_{a<r \leq 2 a} r(m+r) \\
\ll & \log m \cdot \max _{a \ll m} \frac{1}{a} \sum_{m+a<r \leq m+2 a} r(r) \\
\ll & \log m \cdot \max _{a \ll m} \frac{1}{a}\left((m+2 a)^{1 / 2}-(m+a)^{1 / 2}\right) \\
& \quad+\log m \cdot \max _{a \ll m} \frac{1}{a}\left((m+2 a)^{1 / 3}-(m+a)^{1 / 3}\right) \\
& \quad+\log m \cdot \max _{a \ll m} \frac{1}{a} m^{23 / 146} \log ^{3} m \\
\ll & m^{-1 / 2} \log m+T m^{23 / 146-1} \log ^{4} m,
\end{aligned}
$$

if we notice $a \gg m\left(e^{1 / T}-1\right) \gg m / T$.

Inserting (5.8) into (5.7) we get

$$
\begin{aligned}
\Sigma_{2} & \ll \sum_{m \leq X} \frac{r(m)}{m^{2 \sigma-1}}\left(m^{-1 / 2} \log m+T m^{23 / 146-1} \log ^{4} m\right) \\
& \ll X^{1-2 \sigma} \log T+T \log ^{5} T .
\end{aligned}
$$

From (5.4)-(5.9) we get

$$
W_{1} \ll X^{1-2 \sigma} \log T+T \log ^{5} T
$$


Now we estimate $W_{2}$. We have

$$
\text { (5.11) } \begin{aligned}
W_{2}= & \int_{T}^{2 T} d t \int_{X}^{\infty} \int_{X}^{\infty} \frac{F\left(\omega_{1}\right)}{\omega_{1}^{\sigma+1+i t}} \cdot \frac{F\left(\omega_{2}\right)}{\omega_{2}^{\sigma+1-i t}} d \omega_{1} d \omega_{2} \\
= & \int_{X}^{\infty} \int_{X}^{\infty} \frac{F\left(\omega_{1}\right) F\left(\omega_{2}\right)}{\left(\omega_{1} \omega_{2}\right)^{\sigma+1}} d \omega_{1} d \omega_{2} \int_{T}^{2 T}\left(\frac{\omega_{2}}{\omega_{1}}\right)^{i t} d t \\
\ll & \int_{X}^{\infty} \int_{X}^{\infty}\left(\omega_{1} \omega_{2}\right)^{23 / 146-\sigma-1}\left(\log \omega_{1} \log \omega_{2}\right)^{3} \min \left(T, \frac{1}{\left|\log \frac{\omega_{2} \mid}{\omega_{1}}\right|}\right) d \omega_{1} d \omega_{2} \\
\ll & \int_{X}^{\infty} \omega_{1}^{23 / 146-\sigma-1} \log ^{3} \omega_{1} d \omega_{1} \\
& \times \int_{X}^{\omega_{1}} \omega_{2}^{23 / 146-\sigma-1} \log ^{3} \omega_{2} \min \left(T, \frac{1}{\log \frac{\omega_{1}}{\omega_{2}}}\right) d \omega_{2} \\
= & \int_{1}+\int_{2}+\int_{3}
\end{aligned}
$$

where

$$
\begin{aligned}
& \int_{1}=T \int_{X}^{\infty} \omega_{1}^{23 / 146-\sigma-1} \log ^{3} \omega_{1} d \omega_{1} \int_{e^{-1 / T} \omega_{1}}^{\omega_{1}} \omega_{2}^{23 / 146-\sigma-1} \log ^{3} \omega_{2} d \omega_{2}, \\
& \int_{2}=\int_{X}^{\infty} \omega_{1}^{23 / 146-\sigma-1} \log ^{3} \omega_{1} d \omega_{1} \int_{e^{-1 / T} \omega_{1}}^{e^{-1 / 3} \omega_{1}} \omega_{2}^{23 / 146-\sigma-1} \log ^{3} \omega_{2} \frac{1}{\log \frac{\omega_{1}}{\omega_{2}}} d \omega_{2}, \\
& \int_{3}=\int_{X}^{\infty} \omega_{1}^{23 / 146-\sigma-1} \log ^{3} \omega_{1} d \omega_{1} \int_{X}^{e^{-1 / 3} \omega_{1}} \omega_{2}^{23 / 146-\sigma-1} \log ^{3} \omega_{2} \frac{1}{\log \frac{\omega_{1}}{\omega_{2}}} d \omega_{2} .
\end{aligned}
$$

Trivially we have

$$
\int_{3} \ll\left(\int_{X}^{\infty} \omega_{1}^{23 / 146-\sigma-1} \log ^{3} \omega_{1} d \omega_{1}\right)^{2} \ll X^{46 / 146-2 \sigma} \log ^{6} X .
$$

For $\int_{1}$, we have

$$
\begin{aligned}
\int_{1} & \ll T \int_{X}^{\infty} \omega_{1}^{46 / 146-2 \sigma-2} \log ^{6} \omega_{1} d \omega_{1} \int_{e^{-1 / T} \omega_{1}}^{\omega_{1}} d \omega_{2} \\
& \ll T\left(e^{1 / T}-1\right) \int_{X}^{\infty} \omega_{1}^{46 / 146-2 \sigma-1} \log ^{6} \omega_{1} d \omega_{1} \\
& \ll X^{46 / 146-2 \sigma} \log ^{6} X .
\end{aligned}
$$


For $\int_{2}$, we have

$$
\int_{2} \ll \int_{X}^{\infty} \omega_{1}^{46 / 146-2 \sigma-2} \log ^{6} \omega_{1} d \omega_{1} \int_{e^{-1 / 3} \omega_{1}}^{e^{-1 / T} \omega_{1}} \frac{1}{\log \frac{\omega_{1}}{\omega_{2}}} d \omega_{2}
$$

$\ll \int_{X}^{\infty} \omega_{1}^{46 / 146-2 \sigma-2} \log ^{6} \omega_{1} d \omega_{1} \int_{e^{-1 / 3} \omega_{1}}^{e^{-1 / T} \omega_{1}} \frac{\omega_{1}}{\omega_{1}-\omega_{2}} d \omega_{2}$

$\ll \int_{X}^{\infty} \omega_{1}^{46 / 146-2 \sigma-1} \log ^{6} \omega_{1}\left(-\log \left(\omega_{1}-e^{-1 / T} \omega_{1}\right)+\log \left(\omega_{1}-e^{-1 / 3} \omega_{1}\right)\right) d \omega_{1}$

$\ll X^{46 / 146-2 \sigma} \log ^{7} X$.

From (5.11)-(5.14) we get

$$
W_{2} \ll X^{46 / 146-2 \sigma} \log ^{7} X .
$$

Combining (5.3), (5.10) and (5.15) we get

$$
\int_{T}^{2 T}|Z(\sigma+i t)|^{2} d t \ll X^{1-2 \sigma} \log T+T^{2} X^{46 / 146-2 \sigma} \log ^{7} X+T \log ^{5} T .
$$

Now the fourth assertion of Lemma 5.2 follows from (5.16) by taking $\sigma=24 / 73$ and $X=T^{146 / 50}$.

In order to estimate $S_{2}$, we also need the following lemma. Its proof is contained in [7].

LEMMA 5.3. Suppose RH is true. If

$$
\int_{T}^{2 T}|Z(\sigma+i t)|^{2} d t \ll T^{1+\varepsilon}
$$

for some $\sigma \geq 1 / 4$, then

$$
S_{2}=k N^{1 / 2} \sum_{d>y} \frac{\mu(d)}{d^{2}}-k^{\prime} N^{1 / 3} \sum_{d>y} \frac{\mu(d)}{d^{4 / 3}}+O\left(y^{1 / 2}\left(\frac{x}{y^{4}}\right)^{\sigma+\varepsilon}\right)
$$

for $1 \leq y<x^{1 / 4}$.

From Lemmas 5.2 and 5.3 we immediately get

Proposition 5.1. If $R H$ is true, then

$$
S_{2}=k N^{1 / 2} \sum_{d>y} \frac{\mu(d)}{d^{2}}-k^{\prime} N^{1 / 3} \sum_{d>y} \frac{\mu(d)}{d^{4 / 3}}+O\left(N^{24 / 73+\varepsilon} y^{-119 / 146}\right)
$$

for $1 \leq y<N^{1 / 4}$. 
Now we give the proof of the Theorem. Take $y=N^{119 / 768}$. By (2.4), (2.5), (4.7) and Proposition 5.1 we get

$$
\begin{aligned}
L^{\prime}(N)= & k N^{1 / 2} \sum_{d=1}^{\infty} \frac{\mu(d)}{d^{2}}-k^{\prime} N^{1 / 3} \sum_{d=1}^{\infty} \frac{\mu(d)}{d^{4 / 3}} \\
& +O\left(N^{127 / 616} \log ^{963 / 308} N\right),
\end{aligned}
$$

which combined with (2.1) yields the Theorem.

\section{References}

[1] J. Duttlinger und W. Schwarz, Über die Verteilung der Pythagoräischen Dreiecke, Colloq. Math. 43 (1980), 365-372.

[2] M. N. Huxley and W. G. Nowak, Primitive lattice points in convex planar domains, Acta Arith. 76 (1996), 271-283.

[3] J. Lambek and L. Moser, On the distribution of Pythagorean triangles, Pacific J. Math. 5 (1955), 73-83.

[4] H. Menzer, On the number of primitive Pythagorean triangles, Math. Nachr. 128 (1986), 129-133.

[5] W. Müller and W. G. Nowak, Lattice points in planar domains: applications of Huxley's "discrete Hardy-Littlewood method", in: Number-Theoretic Analysis (Vienna, 1988-89), Lecture Notes in Math. 1452, Springer, 1990, 139-164.

[6] W. Müller, W. G. Nowak and H. Menzer, On the number of primitive Pythagorean triangles, Ann. Sci. Math. Québec 12 (1988), 263-273.

[7] W. G. Nowak, Primitive lattice points in starlike planar sets, Pacific J. Math. 170 (1997), 163-178.

[8] C. D. Pan and C. B. Pan, Foundations of the Analytic Number Theory, Science Press, Beijing, 1991 (in Chinese).

[9] J. D. Vaaler, Some extremal problems in Fourier analysis, Bull. Amer. Math. Soc. 12 (1985), 183-216.

[10] R. E. Wild, On the number of primitive Pythagorean triangles with area less than $n$, Pacific J. Math. 5 (1955), 85-91.

Department of Mathematics

Shandong Normal University

Jinan, 250014, Shandong, P.R. China

E-mail: zhaiwg@hotmail.com
Current address:

Graduate School of Mathematics

Nagoya University

Chikusa-ku, Nagoya 464-8602, Japan E-mail: x01002r@math.nagoya-u.ac.jp 